\title{
Comparação entre análises de referência incisal em indivíduos jovens melanodermas brasileiros com oclusão normal
}

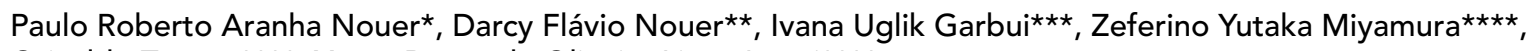

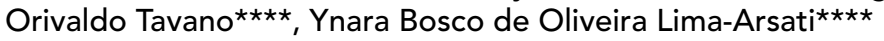

\section{Resumo}

A definição da posição do incisivo inferior oferece ao clínico dados para um correto planejamento e tratamento, assim como melhor estabilidade na pós-contenção. Observa-se, porém, que as análises cefalométricas mais utilizadas em Ortodontia para o diagnóstico geralmente são baseadas em indivíduos leucodermas, que diferem em características craniofaciais das outras raças. Desta forma, o presente trabalho teve como objetivo estudar o comportamento do incisivo inferior em relação às referências incisais preconizadas por Andrade (1-Jr), Interlandi (1-linhaI), Vigorito (1-linhaV) e Ricketts (1-linha AP) em jovens brasileiros melanodermas com oclusão normal, verificando também a ocorrência de dimorfismo sexual. Foram selecionadas 36 telerradiografias de cabeça tomadas em norma lateral de indivíduos brasileiros, melanodermas, na faixa etária de 10 a 14 anos, de ambos os gêneros, com oclusão clinicamente normal e que nunca se submeteram a tratamento ortodôntico, do Setor de Documentação Científica do Curso de Pós-Graduação em Ortodontia da FOP/UNICAMP, nas quais foram traçados os pontos, linhas e planos cefalométricos necessários para obtenção das referências incisais e medidas nas análises sugeridas pelos autores selecionados. Os valores médios obtidos para a posição do incisivo inferior diferiram entre si por duas análises estatísticas, havendo diferença significativa $(\mathrm{p}<0,05)$ entre os métodos estudados. Também não houve dimorfismo sexual. Concluiu-se que a posição dos incisivos inferiores em indivíduos jovens melanodermas brasileiros apresenta inclinação e vestibularização maior que a preconizada pelos autores consultados, e que a análise de referência incisal de Andrade (1-Jr) parece se comportar mais adequadamente para este grupo étnico.

Palavras-chave: Cefalometria. Negros. Grupo ancestral do continente africano. Ortodontia.

* Professor Titular do Programa de Mestrado em Ortodontia do CPO São Leopoldo Mandic, Campinas - SP.

** Professor Titular da Disciplina de Ortodontia - FOP - Unicamp - Piracicaba - São Paulo.

*** Professora Convidada do Curso de Mestrado em Ortodontia do CPO São Leopoldo Mandic - Campinas - SP. Doutoranda em Ortodontia - FOP/Unicamp - Piracicaba - São Paulo.

**** Professor Titular do Programa de Mestrado em Ortodontia do CPO São Leopoldo Mandic, Campinas - SP. 


\section{INTRODUÇÃO}

Com o advento da cefalometria por Broadbent ${ }^{6}$, os parâmetros craniofaciais puderam ser analisados com mais facilidade, mas a partir da década de 50 , os ortodontistas começaram a observar que tanto o diagnóstico quanto o resultado terapêutico eram limitados pelo tipo facial do paciente, o qual variava consideravelmente de acordo com o grupo étnico.

Cotton et al. ${ }^{7}$, utilizando a análise de Downs, observaram que os negros americanos apresentavam protrusão da maxila, perfil convexo, plano mandibular inclinado e incisivos mais inclinados que os caucasianos. Steiner ${ }^{21}$ admitiu que suas normas, que deveriam ser tomadas como referência inicial, podiam ser modificadas por alguns fatores, como raça e gênero. Estes achados alertaram os ortodontistas de que as mesmas normas cefalométricas não poderiam ser usadas para populações de etnias diferentes ${ }^{5}$.

Altemus $^{2}$ verificou que os incisivos inferiores eram mais vestibularizados em melanodermas americanos. No estudo de Kowalski et al. ${ }^{15}$, o aspecto mais singular foi a distância do incisivo inferior à linha NB (supramental), demonstrando que a protrusão dos incisivos inferiores era muito maior nos negros americanos. Jacobson ${ }^{14}$ encontrou um padrão semelhante na inclinação vestibular dos incisivos inferiores em melanodermas sul-africanos. A protrusão dentária bimaxilar em afro-americanos também foi um achado de Drummond ${ }^{9}$; Fonseca, Klein ${ }^{11}$; Alexander e Hitchcock ${ }^{1}$. Mais recentemente Anderson et al. ${ }^{3}$ encontraram protrusão dos incisivos inferiores em indivíduos com oclusão normal e Dandajena e Nanda ${ }^{8}$ confirmaram estes achados por meio de múltiplas referências incisais $\left(\mathrm{IMPA}=105.8^{\circ} \pm 6.0^{\circ}, \mathrm{L} 1-\mathrm{APog}=6.9^{\circ} \pm 2.7^{\circ}\right.$, $\mathrm{L} 1-\mathrm{NB}=37.6^{\circ} \pm 4.9^{\circ}$ )

Em âmbito nacional, autores têm estudado relações cefalométricas em indivíduos melanodermas brasileiros com oclusão normal4,10,16,18. Também Medeiros ${ }^{17}$ observou que melanodermas do gênero feminino apresentavam acentuadas protrusão e inclinação vestibular dos incisivos, principalmente dos inferiores, quando comparado ao grupo leucoderma. Fortes ${ }^{12}$ encontrou aspecto morfológico típico de maior protrusão de incisivos superiores e inferiores.

Desta forma, torna-se aparente que as normas cefalométricas existentes para o posicionamento ideal e equilibrado dos incisivos inferiores preconizadas por Ricketts ${ }^{20}$; Interlandi ${ }^{13}$; Vigorito ${ }^{22}$; Andrade (apud NOUER et al. ${ }^{19}$, 1996) não poderiam ser aplicadas aos melanodermas, uma vez que foram tomadas de indivíduos leucodermas.

\section{PROPOSIÇÃO}

Embora vários estudos tenham sido conduzidos para determinar as diferenças craniofaciais entre os grupos étnicos, as informações quanto aos padrões cefalométricos para melanodermas brasileiros são limitadas. Assim, fica clara a necessidade de pesquisas que permitam um diagnóstico mais acurado para estes indivíduos. Este trabalho se propôs a:

1) estudar o comportamento do incisivo inferior em relação às referências incisais nas técnicas de Andrade (Jr), Interlandi (I), Vigorito (V) e Ricketts (AP) em pacientes melanodermas com oclusão normal;

2) comparar estas medidas com aos parâmetros considerados normais para leucodermas;

3) comparar o comportamento das grandezas entre si;

4) verificar a ocorrência de dimorfismo sexual.

\section{MATERIAL E MÉTODO}

\section{Material}

Para a realização do presente estudo, selecionaram-se 36 telerradiografias em norma lateral, provenientes do arquivo do Setor de Documentação Científica do Curso de Pós-Graduação em Ortodontia da Faculdade de Odontologia de Piracicaba, Universidade Estadual de Campinas. As telerradiografias foram tomadas de indivíduos 
brasileiros, melanodermas, na faixa etária de 10 a 14 anos, de ambos os gêneros, com oclusão clinicamente normal e que nunca haviam se submetido a tratamento ortodôntico.

\section{Método}

As telerradiografias foram divididas de acordo com o gênero e os traçados cefalométricos feitos por um único pesquisador, em sala escura e sobre um negatoscópio.

O traçado foi confeccionado sobre papel ultraphan de tamanho padronizado de $17,5 \times 17,5 \mathrm{~cm}$ e $0,07 \mathrm{~mm}$ de espessura, preso com fita adesiva sobre a telerradiografia, utilizando lapiseira com grafite $0,5 \mathrm{~mm}$ tipo $\mathrm{HB}$, régua transparente com subdivisões de $0,5 \mathrm{~mm}$, esquadro e template. Foram delimitadas as estruturas anatômicas do crânio e da face, e os pontos cefalométricos necessários para a obtenção das referências incisais e medidas nas análises sugeridas pelos autores: Andrade (apud NOUER et al. ${ }^{19}$, 1996); Vigorito ${ }^{22}$; Ricketts $^{20}$ e Interlandi ${ }^{13}$.

Os pontos e as grandezas cefalométricas utilizados nesta pesquisa estão descritos no quadro 1 .
Os desenhos anatômicos com os pontos, linhas e planos das diferentes análises podem ser visualizados nas figuras 1, 2, 3 e 4 .

De posse dos resultados, obtiveram-se as médias, desvio-padrão, os valores máximo e mínimo, e a ocorrência de dimorfismo sexual. Para descrever as diferenças na amostra, foi realizada análise estatística de Mann-Whitney ao nível de 5\% por não haver distribuição normal na amostra, e para a comparação das grandezas selecionadas entre si foi utilizado teste de Friedman e o não paramétrico de comparações múltiplas, sendo os mesmos utilizados para comparação de mais de dois itens em amostra sem distribuição normal.

\section{RESULTADOS}

Os resultados da pesquisa podem ser visualizados nas tabelas 1, 2, 3, 4 e 5 e nos gráficos 1, 2, 3 e 4 .

\section{DISCUSSÃO}

A maioria dos autores que estudaram as relações cefalométricas em indivíduos melanodermas brasileiros com oclusão normal detectaram uma

\begin{tabular}{|c|c|}
\hline Ponto A & Ponto mais profundo da curvatura anterior da maxila \\
\hline Ponto B & Ponto localizado na maior profundidade da curvatura anterior da sínfise \\
\hline Cjr & $\begin{array}{l}\text { Centro do raio que define o arco de abertura e fechamento que passa simultaneamente pelos pontos } A \text { e } B \text {, localizado na corti- } \\
\text { cal distal do ramo da mandíbula }\end{array}$ \\
\hline $\operatorname{Arco~Jr}$ & Arco que passa simultaneamente pelos pontos A e B e define a localização do ponto Jr quando cruza com o plano oclusal \\
\hline Ponto Jr & Referência incisal situada no entrecruzamento do plano oclusal com o arco Jr \\
\hline Ponto V & $\begin{array}{l}\text { Ponto localizado na metade da distância das corticais vestibular e lingual da sínfise mandibular em uma linha paralela ao plano } \\
\text { mandibular que parte do ponto } E\end{array}$ \\
\hline Ponto E & Ponto obtido a partir de uma perpendicular ao plano mandibular que tangencia a sínfise mandibular \\
\hline Ponto N & Ponto mais anterior da sutura frontonasal \\
\hline Ponto p' & Ponto localizado na intersecção da linha NA com o assoalho da fossa nasal \\
\hline Linha I & Referência incisal traçada do ponto p' ao ponto $E$, representada por um segmento de $1 \mathrm{~cm}$ cruzando o plano oclusal \\
\hline Ponto Pg & Ponto mais anterior da sínfise mandibular \\
\hline Linha AP & Referência incisal representada por uma linha unindo os pontos $A$ ao $\mathrm{Pg}$ \\
\hline Ponto Me & Ponto mais inferior da sínfise mandibular \\
\hline Ponto Go & $\begin{array}{l}\text { Ponto localizado no ângulo da mandíbula e obtido pelas linhas tangentes à borda posterior do ramo e à borda inferior do corpo } \\
\text { da mandíbula. Traça-se a bissetriz do ângulo formado entre as linhas e marca-se na cortical mandibular }\end{array}$ \\
\hline
\end{tabular}

Quadro 1 - Pontos e grandezas cefalométricas utilizados no estudo. ${ }^{13,19-22}$ 


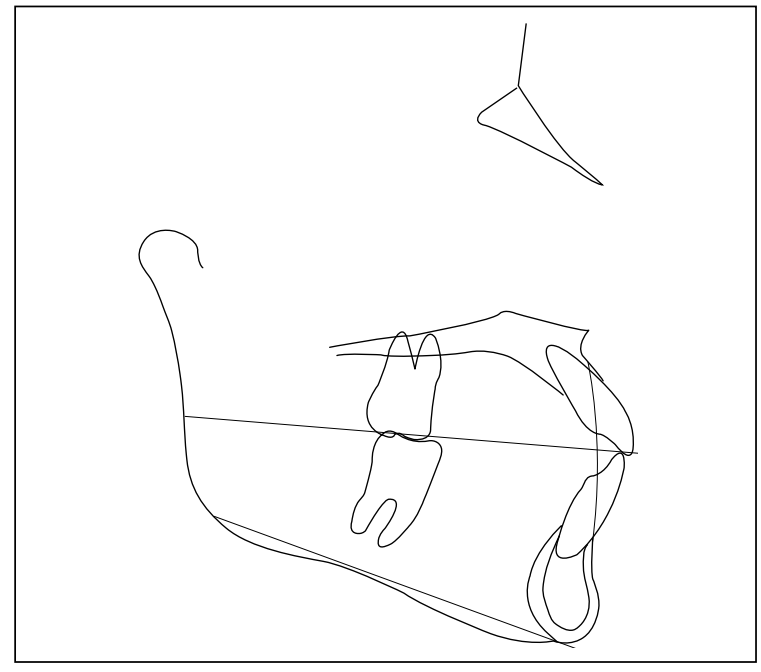

FIGURA 1 - Análise de Andrade (1-Jr).

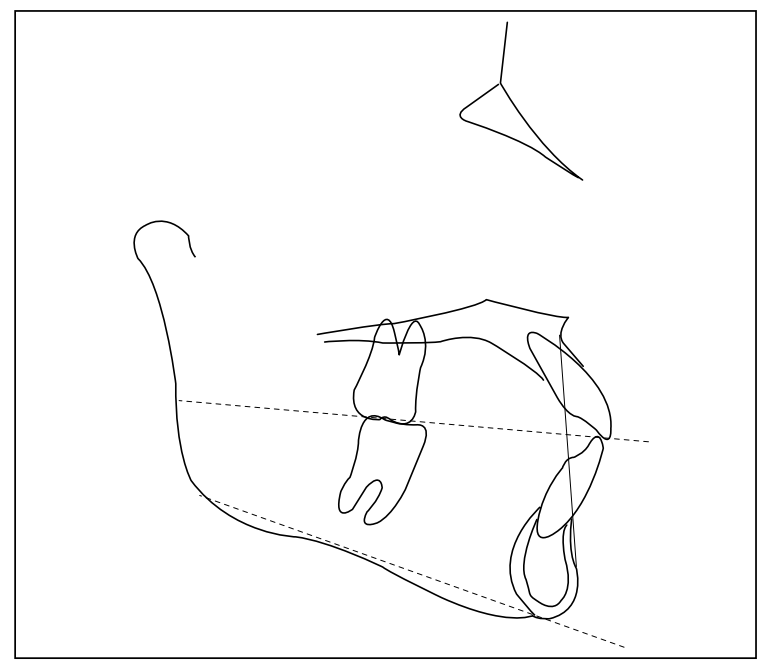

FIGURA 3 - Análise de Ricketts (1-AP).

significativa proclinação das bases apicais ${ }^{4,10,12,16,}$ ${ }^{17,18}$. No entanto, não existem estudos que comparem e busquem análises cefalométricas que melhor se adaptem a este padrão racial diferenciado.

Pelas tabelas 1, 2, 3, 4, verifica-se que as médias diferem das estabelecidas em caucasianos como norma nas análises de seus respectivos autores. De fato, os valores negativos demonstram grande inclinação vestibular dos incisivos inferiores, sendo menor na análise de Andrade (Tab. 2, Gráf. 2) e maior na de Ricketts (Tab. 3, Gráf. 3). Nesta última análise, Ricketts preconiza que a borda incisal

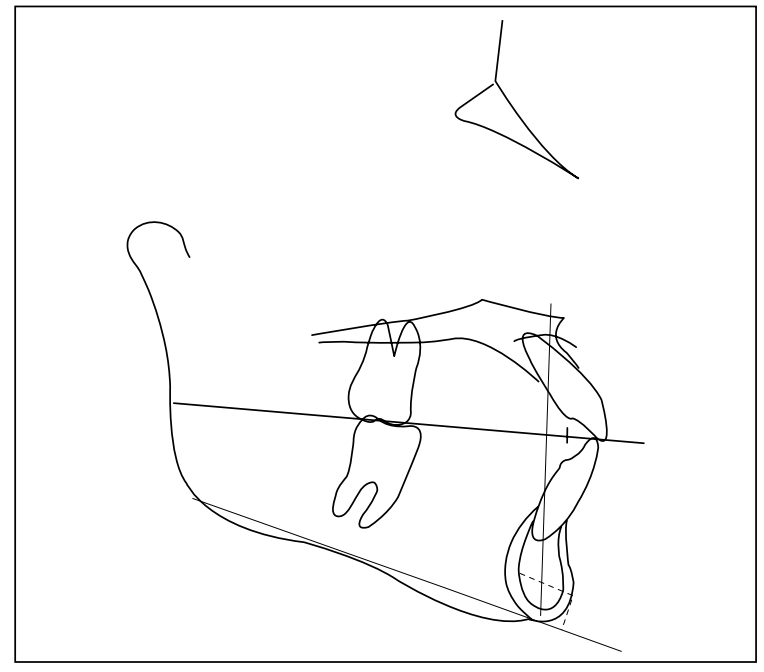

FIGURA 2 - Análise de Vigorito (1-Linha V).

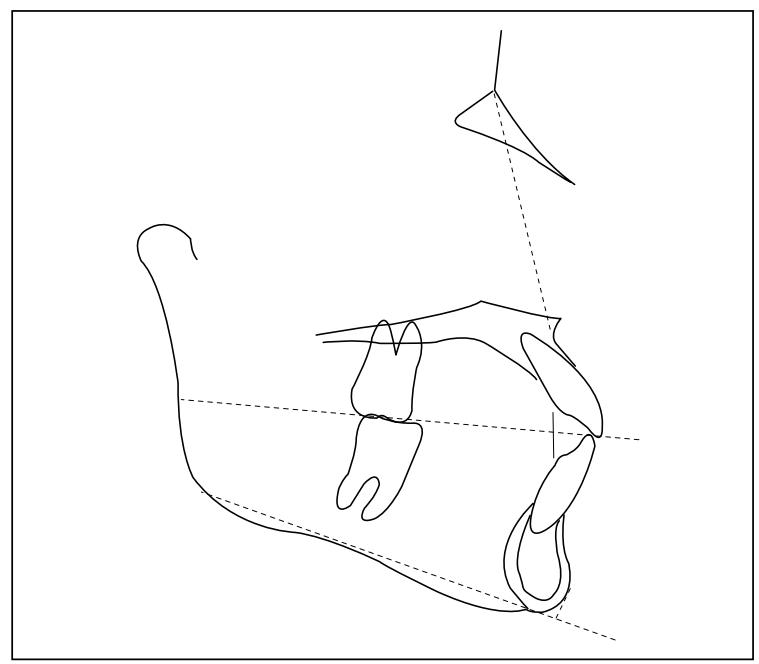

FIGURA 4 - Análise de Interlandi (1-Linha I).

deve se localizar $0,5 \pm 2,7 \mathrm{~mm}$ à esquerda da linha AP, porém, nos indivíduos melanodermas, a posição de normalidade foi alterada para $-5,90 \mathrm{~mm}$ em média. De acordo com Interlandi, a posição normal dos incisivos inferiores é identificada quando a linha I coincide com o limite lingual da borda incisal. Com relação às outras duas análises, Vigorito (1-linha V) e Andrade (1-Jr), a borda incisal do incisivo inferior deve coincidir com as linhas obtidas a partir dos traçados pertencentes a cada método. Em todos os casos, os valores apresentados pela aplicação dessas análises são negativos, 
Tabela 1 - Média, desvio-padrão, valor máximo e mínimo da referência incisal (V).

\begin{tabular}{cccc}
\hline & \multicolumn{2}{c}{ Gênero } & \multirow{2}{*}{ Média } \\
\cline { 2 - 3 } & Feminino & Masculino & \\
\hline Média & $-4,9000^{\mathrm{a}}$ & $-6,1563^{\mathrm{a}}$ & $-5,4583$ \\
Desvio & 2,2511 & 3,6593 & 2,9817 \\
Mínimo & $-10,000$ & $-16,000$ & $-16,000$ \\
Máximo & $-0,5000$ & 0,5000 & 0,5000 \\
\hline
\end{tabular}

${ }^{a}$ não diferem entre si pelo teste de Mann-Whitney $(p>0,05)$.

Tabela 2 - Média, desvio-padrão, valor máximo e mínimo da referência incisal (Jr).

\begin{tabular}{cccc}
\hline & \multicolumn{2}{c}{ Gênero } & \multirow{2}{*}{ Média } \\
\cline { 2 - 3 } & Feminino & Masculino & \\
\hline Média & $-2,7750^{\mathrm{a}}$ & $-3,4688^{\mathrm{a}}$ & $-3,0833$ \\
Desvio & 1,6016 & 2,3697 & 1,9803 \\
Mínimo & $-6,0000$ & $-10,000$ & $-10,000$ \\
Máximo & 0,0000 & 1,0000 & 1,0000 \\
\hline
\end{tabular}

${ }^{a}$ não diferem entre si pelo teste de Mann-Whitney $(p>0,05)$.

Tabela 3 - Média, desvio padrão, valor máximo e mínimo da referência incisal (AP).

\begin{tabular}{cccc}
\hline & \multicolumn{2}{c}{ Gênero } & \multirow{2}{*}{ Média } \\
\cline { 2 - 3 } & Feminino & Masculino & \\
\hline Média & $-5,4250^{\mathrm{a}}$ & $-6,5000^{\mathrm{a}}$ & $-5,9028$ \\
Desvio & 2,2200 & 3,5214 & 2,8780 \\
Mínimo & $-10,000$ & $-16,000$ & $-16,000$ \\
Máximo & $-1,0000$ & 0,0000 & 0,0000 \\
\hline
\end{tabular}

${ }^{a}$ não diferem entre si pelo teste de Mann-Whitney $(p>0,05)$.

Tabela 4 - Média, desvio padrão, valor máximo e mínimo da referência incisal (I).

\begin{tabular}{cccc}
\hline & \multicolumn{2}{c}{ Gênero } & \multirow{2}{*}{ Média } \\
\cline { 2 - 3 } & Feminino & Masculino & \\
\hline Média & $-5,2000^{\mathrm{a}}$ & $-6,4063^{\mathrm{a}}$ & $-5,7361$ \\
Desvio & 2,3530 & 3,3971 & 2,8847 \\
Mínimo & $-9,5000$ & $-15,000$ & $-15,000$ \\
Máximo & 0,0000 & 0,5000 & 0,5000 \\
\hline
\end{tabular}

a não diferem entre si pelo teste de Mann-Whitney $(p>0,05)$.

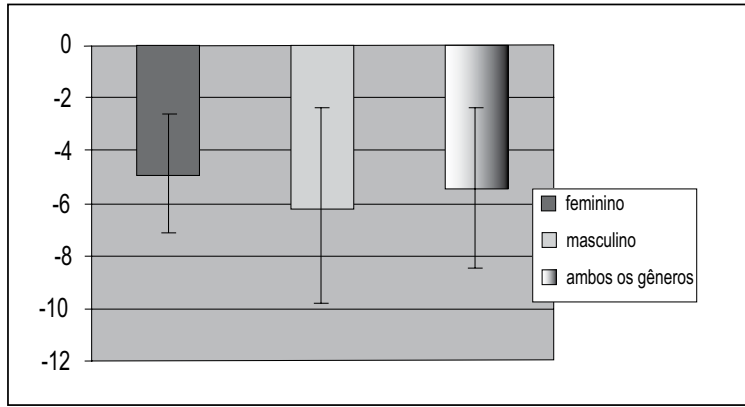

GRÁFICO 1 - Média e desvio-padrão da referência incisal (V).

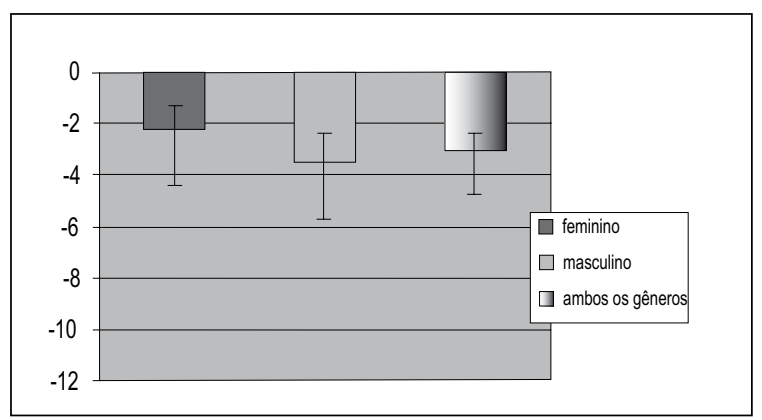

GRÁFICO 2 - Média e desvio-padrão da referência incisal (Jr).

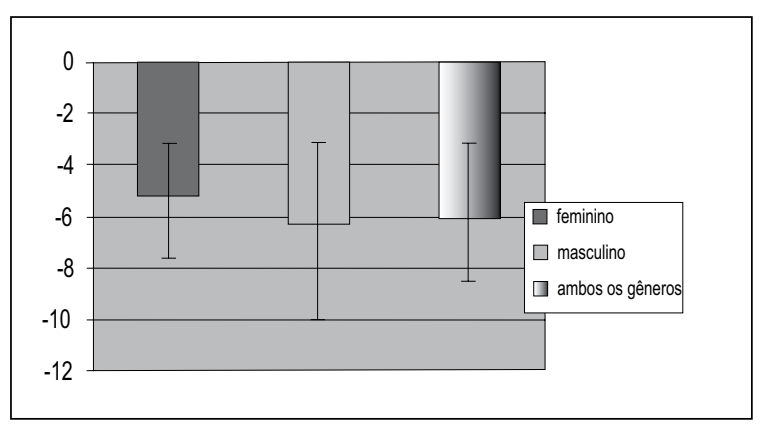

GRÁFICO 3 - Média e desvio padrão da Referência incisal (AP).

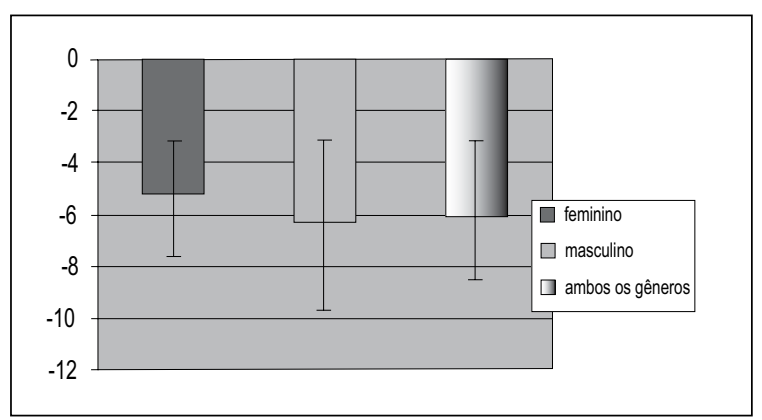

GRÁFICO 4 - Média e desvio padrão da referência incisal (I). 
Tabela 5 - Comparação entre as análises.

\begin{tabular}{ccc}
\hline Método & Posto total & Posto médio \\
\hline V & 90,5000 & $2,5139^{\mathrm{b}}$ \\
$\mathrm{Jr}$ & 143,0000 & $3,9722^{\mathrm{a}}$ \\
$\mathrm{AP}$ & 55,5000 & $1,5417^{\mathrm{c}}$ \\
$\mathrm{I}$ & 71,0000 & $1,9722^{\mathrm{bc}}$ \\
\hline
\end{tabular}

* Médias seguidas de letras distintas na vertical diferem entre si pelo teste de Friedman e teste não paramétrico de comparações múltiplas.

ou seja, os incisivos inferiores se posicionaram à frente da linha incisal, sendo estes valores considerados normais para este grupo étnico.

A proclinação dos incisivos inferiores nas análises de referência incisal deste estudo concorda com vários outros autores ${ }^{12,14,15,18}$ e reforça a necessidade de valores de normalidade diferentes dos preconizados inicialmente.

É interessante notar que na análise de Andrade, os valores obtidos foram em média duas vezes menores que nas outras análises (Tab. 5, p<0,05). Isto significa que a referência incisal 1-Jr é mais adequada em relação às bases apicais mais proclinadas dos melanodermas, adotando como normalidade uma inclinação vestibular ligeiramente maior, em concordância com o estudo realizado por Nouer et al. ${ }^{19}$

Em todas as referências incisais avaliadas $(\mathrm{V}, \mathrm{Jr}$, I, AP), notou-se claramente a ausência de dimorfismo sexual, confirmando a proposta de um valor de normalidade invariável.

\section{CONCLUSÃO}

A análise de referência incisal de acordo com Vigorito, Andrade, Ricketts e Interlandi em indivíduos melanodermas revelou que as posições dos incisivos inferiores apresentaram valores negativos e diferentes dos valores de normalidade em leucodermas preconizados pelos autores. Neste estudo, especialmente na análise de Andrade, os valores obtidos foram, em média, duas vezes menores que nas outras análises, levando-nos a concluir que o comportamento desta análise é mais adequado para o grupo étnico avaliado, por adotar como normalidade uma inclinação vestibular maior. Em todas as referências incisais avaliadas (V, Jr, I, AP), notou-se claramente a ausência de dimorfismo sexual.

\title{
Comparison among incisal landmark analysis of black brazilian youngsters with normal occlusion
}

\begin{abstract}
Defining the position for the mandibular incisor provides data for a correct treatment planning and treatment itself, in order to achieving a better stability after retention. However, it has been observed that cephalometric analyses that are usually employed in orthodontics for diagnosis are based on studies on caucasians, whose craniofacial characteristics differ from those of other races. Thus, the present study aims to investigate the behavior of mandibular incisor related to incisal landmarks that are commended by Andrade (1-Jr), Interlandi (1- I line), Vigorito (1- V line) and Ricketts (1-APo line), in african-american children with normal occlusion, and to verify the occurrence of sexual dimorphism. Thirty-six lateral skull films from african-american subjects were selected from the FOP/UNICAMP Department of Orthodontics Post-Graduation Program Scientific Recordings; the patients ages varied from 10 to 14 years, and they presented clinically excellent occlusion, and have not undergone orthodontic treatment. The cefalogram required for the suggested analyses were drawed. The presented values for the mandibular incisor position differ among themselves as by two different statistical analysis; there is also significant difference among the referred methods. No sexual dimorphism was found in this study. It was shown that the position of mandibular incisor of african-american children presented greater labioversion than the same parameters as commended by the proposed authors, and that Andrade analysis seemed more adequate to this ethnic group.
\end{abstract}

Key words: Cephalometry. Black people. African continental ancestry group. Orthodontics. 


\section{REFERÊNCIAS}

1. ALEXANDER, T. L.; HITCHCOCK, H. P. Cephalometric standards for American Negro children. Am J Orthod, St. Louis, v. 74, p. 298-304, 1978.

2. ALTEMUS, L. A. A comparison of cephalofacial relationships. Angle Orthod, Appleton, v. 30, p. 228-239, 1960

3. ANDERSON, A. A.; ANDERSON, A. C.; HORNBUCKLE, A. C.; HORNBUCKLE, K. Biological derivation of a range of cephalometric norms for children of African American descent (after Steiner). Am J Orthod Dentofacial Orthop, St. Louis, v. 118, p. 90-100, 2000.

4. ARAÚJO, M. B. B.; SAKIMA, T. Comparação cefalométrica entre negróides e caucasóides. RGO, Porto Alegre, v. 37, p. 225-228, 1989.

5. BERTOZ, F. A.; MARTINS, D. R. Determinação da linha "I" em melanodermas brasileiros, masculinos de 12 a 17 anos, com oclusão normal. Ortodontia, São Paulo, v. 14, n. 3, p. 186-198, 1981.

6. BROADBENT, B. H. A new x-ray technique and its application to Orthodontics. Angle Orthod, Appleton, v. 1, p. 45-66, 1931

7. COTTON, W. N.; TAKANO, W. S.; WONG, W. W.; WYLIE, W. L. Downs analysis applied to three other ethnic groups. Angle Orthod, Appleton, v. 21, p. 213-220, 1951.

8. DANDAJENA, T. C.; NANDA, R. S. Bialveolar protrusion in a Zimbabwean sample. Am J Orthod Dentofacial Orthop, St. Louis, v. 123, p. 133-137, 2003.

9. DRUMMOND, R. A. A determination of cephalometric norms for the negro race. Am J Orthod, St. Louis, v. 54, p. 670-682, 1968.

10. ELY, M. R.; ANTUNES, V. M.; OLIVEIRA, M. G. Estudo de cinco dimensões esqueléticas lineares, em indivíduos da raça negra, através de análise cefalométrica. Rev Odontol Univ São Paulo, São Paulo, v. 13, p. 401-405, 1999.

11. FONSECA, R. J.; KLEIN, W. D. A cephalometric evaluation of American Negro women. Am J Orthod, St. Louis, v. 73, p. 152-160, 1978.
12. FORTES, L. A. P. Avaliação de medidas cefalométricas de indivíduos negros, brasileiros, portadores de oclusão excelente. 2000. 161 f. Dissertação (Mestrado) - Faculdade de Odontologia, Universidade Federal do Rio de Janeiro, Rio de Janeiro, 2000

13. INTERLANDI, S. Linha I na análise morfodiferencial para o diagnóstico ortodôntico. Rev Fac Odontol São Paulo, São Paulo, v. 9, p. 289-310, 1971.

14. JACOBSON, A. The craniofacial pattern of the South African Negro. Am J Orthod, St. Louis, v. 73, p. 681-691, 1978.

15. KOWALSKI, C. J.; NASJLETI, C.; WALKER, G. F. Differential diagnosis of American adult male black and white populations using Steiner's analysis. Angle Orthod, Appleton, v. 44 p. 346-350, 1974.

16. MAGNANI, M. B. B. A.; ALMEIDA, M. H. C.; SIQUEIRA, V. C. V. Análise de "Wits" em indivíduos negróides. Rev Bras Odontol, Rio de Janeiro, v. 47, p. 52-60, 1990.

17. MEDEIROS, M. A. Q. B. Estudo cefalométrico do padrão dentário de jovens melanodermas brasileiros do sexo feminino com "oclusão normal". 1986. Dissertação (Mestrado) - Faculdade de Odontologia de Bauru, Universidade de São Paulo, Bauru, 1986.

18. MORAES, C. Cefalometria: determinação do padrão esquelético das adolescentes melanodermas brasileiras, com "oclusão normal". 1986. Dissertação (Mestrado)-Faculdade de Odontologia de Bauru, Universidade de São Paulo, Bauru, 1986.

19. NOUER, P. R. A.; MARINHO FILHO, A. V.; EL-GUINDY JÚNIOR M. M. Uma nova proposta de mensuração de referência incisal através da imagem do ramo ascendente e dos pontos $A$ e $B$ em telerradiografia lateral. Rev Paul Odontol, São Paulo, v. 18, p. 14-22, 1996

20. RICKETTS, R. M. A foundation for cephalometric communication. Am J Orthod, St. Louis, v. 46, p. 330-357, 1960.

21. STEINER, C. C. Cephalometric for you and me. Am J Orthod, St. Louis, v. 39, p. 729-755, 1953.

22. VIGORITO, J. W. Proposição de uma análise cefalométrica para o diagnóstico e plano de tratamento ortodôntico. Ortodontia, São Paulo, v. 7, p. 141-152, 1974
Endereço para correspondência

Paulo Roberto Aranha Nouer

Rua Américo de Moura, 45

Campinas /SP

E-mail: nouerp@gmail.com 